\title{
Towards self-powered and autonomous wearable glucose sensor
}

\author{
Sara S. Ghoreishizadeh ${ }^{1}$, Despina Moschou ${ }^{2}$, Dearbhla McBay ${ }^{2}$, \\ Carla Gonalez-Solino ${ }^{3}$, Gorachand Dutta ${ }^{2}$, Mirella Di Lorenzo ${ }^{3}$, and Ahmed Soltan ${ }^{4}$ \\ ${ }^{1}$ Dept. of Electronic \& Electrical Engineering, Imperial College London, UK \\ ${ }^{2}$ Centre for Biosensors, Bioelectronics and Biodevices, Dept. of Electronic \& Electrical Eng., Uni. of Bath, UK \\ ${ }^{3}$ Centre for Biosensors, Bioelectronics and Biodevices, Dept. of Chemical Engineering, Uni. of Bath, UK \\ ${ }^{4}$ School of Eng., Newcastle University, Newcastle upon Tyne,UK
}

Email: s.ghoreishizadeh14@imperial.ac.uk,d.moschou@bath.ac.uk, Ahmed.Abd-El-Aal@newcastle.ac.uk

\begin{abstract}
Blood glucose diagnostic systems are a world-wide success story. Nevertheless, all the painless solutions available are too expensive to be disposable. We aim to bridge this gap by developing a painless disposable diabetes diagnostic patch. Our envisaged device is fully integrated and autonomous: harvests the required energy from the environment and features sensor autocalibration in real-time. In this paper, we present the design and preliminary results of the different parts in the wearable patch: electrochemical glucose sensor developed on scalable PCB technology, biofuel cell based on glucose in the biofluid sample, and instrumentation electronics designed on a PCB with energy and data management blocks.
\end{abstract}

Index Terms-biofuel cell, wearable sensor technology, PCB instrumentation, sensor calibration.

\section{INTRODUCTION}

Worldwide, millions of patients suffer from chronic disease such as asthma, diabetes and cardiovascular conditions. Diabetes mellitus is an important chronic disease affecting increasing more than 300 million people worldwide [1]. The World Health Organization (WHO) has placed diabetes among the top 10 world diseases with highest mortality rates. Until now, diabetes has been considered a disease of predominantly rich nations; this is not the case anymore. Diabetes prevalence has been rising more rapidly in low and middle income countries. Furthermore, failure of continuous monitoring of glucose leads for some case to serious health issues and sometimes to death.

While blood glucose diagnostic systems at the Point of Care are a world-wide success story, all the painless solutions (i.e. not requiring finger pricking to extract blood) available are too expensive (ca. $£ 60$ per sensor) to be disposable and suitable for a mass population preventative screening in low and middle income countries. Another limitation of the point of care technology is that the they mostly use lithium batteries as energy source [2]. Indeed, these batteries present a strong challenge for the disposable sensors because of their price and their non-recyclable nature. On the other hand, glucose can be used to generate energy by building enzymatic fuel cells [3][5]. The generated energy from the biofuel cell may be even enough to power the circuits for the sensor with the current advances in the technology.
The non-idealities of chemical sensors such as wear (due to loss of enzyme activity) and instability (due to for instance occlusion and non-reversible non-specific binding) leads to loss of sensitivity or time-varying sensitivity. This necessitates frequent sensor re-calibrations to find the exact sensitivity prior to interpreting the measured signal of the sensor. The traditional calibration methods are based on parallel measurements with a gold standard and corrections based on retrospective forecasts [6]. For example, the CGM sensors typically require two calibrations per day with finger prick tests, imposing extra cost and inconvenience to the user.

A wearable glucose sensor technology that is cheap, painfree, autonomous, and is powered from green energy sources makes a breakthrough in diabetes screening and monitoring. Such a system will use scalable technology ,for instance Printed-circuit-board (PCB) or semiconductor technology, to benefit from the economies of scale.

In this work we present the preliminary results towards the development of such a system. The conceptual diagram of the patch is shown Fig. 1. The idea is that the system sends the readings to a smartphone every 10 s of minutes. We show the integration of the sensor with PCB (Section II) and discuss a possible method for self-calibration of the sensor based on a built-in impedance spectroscopy. We exploit, for the first time covalent immobilization of Glucose oxidase on PCB electrodes for energy harvesting from the biofluid (Section III). The idea here is to extract energy from the same fluid using a

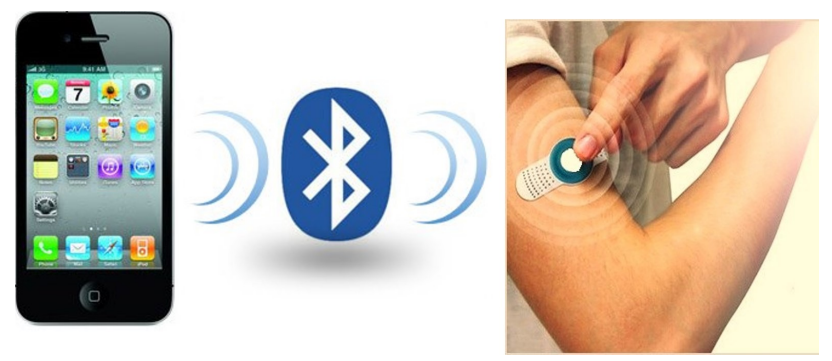

Fig. 1. Conceptual diagram of the proposed system which shows the sensor patch and communicating to mobile application through Bluetooth 
biofuel cell embedded in the patch. Furthermore, we describe a wireless embedded system comprising instrumentation and data and power management modules (Section IV).

\section{SYSTEM DESIGN}

The system comprises of a glucose sensor that senses the glucose levels in the interstitial fluid. The fluid is extracted from under the skin using microneedles and directed, using a microfluidics system, towards the glucose sensor and then passes on top of a biofuel cell before it is collected within a reservoir on the patch where it may evaporate. The biofuel cell generates energy by oxidising the glucose in the fluid. This energy is collected and stored on the device and later used to run the instrumentation and data transmission. The power management mode works based on the event driven technique [7], [8] to optimize the usage of the generated energy. The sensor, biofuel cell and the electronics are presented in the next sections. The microfluidics and microneedle design will be presented in future publications.

\section{GLuCOSE SENSOR ON PCB}

The sensor is designed based on electrochemical sensing of glucose coupled with amperometric measurement. The sensor is developed by covalently immobilized glucose oxidase (GOx) on the commercially fabricated PCB electrode surface. The details of sensor fabrication are presented in [9] and briefly explained here.

The sensor electrodes were designed in PCB CAD software (Altium) and commercially fabricated in a commercial PCB manufacturing facility. The copper electrodes on the fabricated PCB were electroplated with a hard-gold finish in order to exploit the pore-free deposition and low contact resistance achieved by this technique. The gold-plated electrodes were exploited as working, counter and reference electrodes. After cleaning the electrodes [9], a self-assembled monolayer (SAM) of MPA (3 Mercaptopropoinic acid) was formed on the electrode surface by immersing the electrodes in an ethanol solution containing MPA. Then a drop of PBS containing glucose oxidase was dispensed onto the electrode, incubated overnight at $4{ }^{\circ} \mathrm{C}$. A calibration plot of the chronoamperometric current at $60 \mathrm{sec}$ is shown in Fig. 2 where each data point represents three separate measurements using new electrodes. The average sensitivity of the sensor is $150 \mathrm{nA} / \mathrm{mM}$.

\section{A. Sensor Calibration}

The authors have studied the electrochemical impedance and the sensitivity of commercially available CGM sensors (Medtronic Enlite sensors; working electrode area of $2 \mathrm{~mm} 2$ ) over the life-time of the sensors. The results presented in [10] show that the sensitivity is related to the double-layer capacitance and charge-transfer resistance, based on results acquired from a sensor that showed substantial sensitivity drop. Two data clusters are extracted that relate the sensor sensitivity to its impedance before and after the sensitivity drops by more than $50 \%$ (see Fig. 3). These indicate that the variations in the sensor capacitance may be used as an

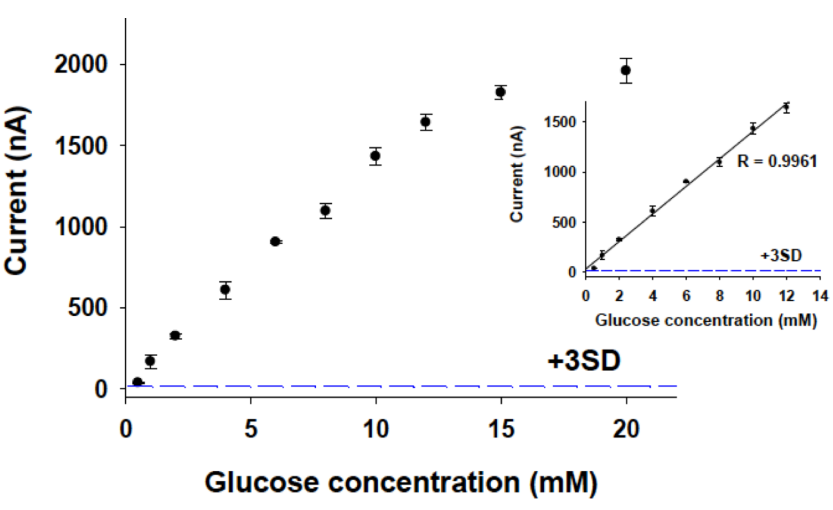

Fig. 2. Calibration plot of glucose concentration based on chronoamperometric currents at $60 \mathrm{sec}$. Each data point represents the mean standard deviation (SD) of three separate measurements obtained using new sensors. Inset shows a magnified view of the data points at $500 \mu \mathrm{M}$ to $12 \mathrm{mM}$. Reprinted from [9]

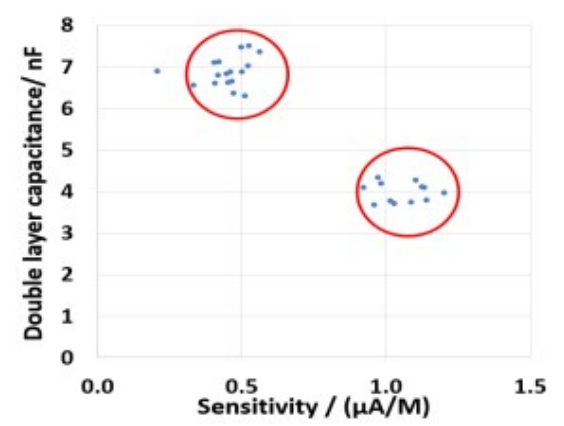

Fig. 3. The correlation between sensor sensitivity and double layer capacitance: two data clusters are visible around the initial and reduced sensitivity of the sensor. Reprinted from [10]

indication of the end of the life-time of the sensor. This feature can be embedded into the wearable device to check the health of the sensor and to implement real-time calibration based on impedance; eliminating the need for external gold references for sensor calibration. Our ongoing studies are on fully modeling the sensitivity-impedance correlation in the custom-designed sensors presented in the previous section.

\section{BIOFUEL CELL}

A two-electrode fuel cell is designed and fabricated on PCB. The Cathode and Anode electrodes are developed through simple cleaning and post-processing steps of the fabricated PCB. The material and methods as well as preliminary results are presented here.

\section{A. materials and methods}

Ammonium hydroxide, hydrogen peroxide, 3Mercaptopropoinic acid, glucose oxidase, glucose and other reagents for buffer solutions were purchased from Sigma-Aldrich.

The fuel cell electrodes were designed and fabricated in a commercial PCB manufacturing facility as illustrated in Fig. 5. The copper electrodes were electroplated with a hard-gold 


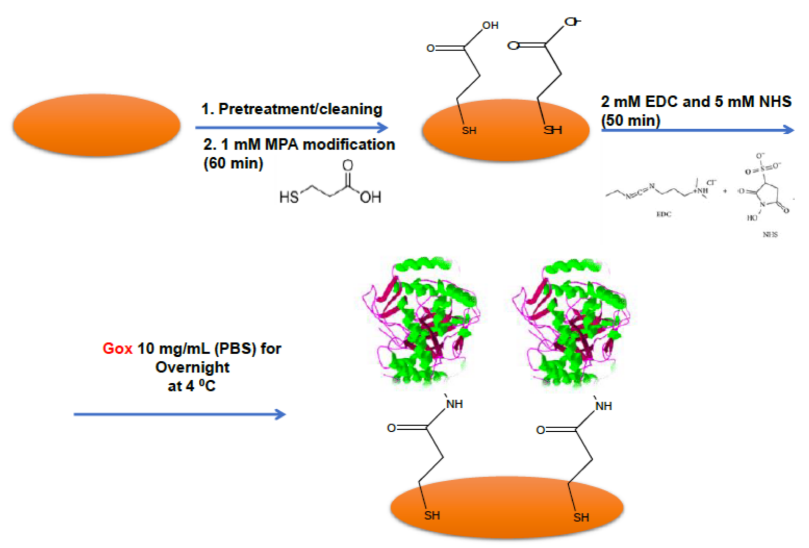

Fig. 4. Schematic illustration of the GOx immobilization on PCB surface. Reprinted from [9].

finish. All finished PCBs were thoroughly cleaned to remove organic contamination prior to any surface modification (15 sonication in acetone, 15 sonication in ethanol and subsequent hydrogen peroxide, ammonium hydroxide and Milli-Q water in a $1: 1: 5$ ratio).

\section{B. Electrode development}

A self-assembled monolayer (SAM) of MPA (3Mercaptopropoinic acid) was formed on the pre-cleaned PCB gold to form the anode electrode (Fig. 5 (a)) by immersing in an ethanol solution containing $1 \mathrm{mM}$ MPA for $1 \mathrm{~h}$ at RT followed by thoroughly rinsing with ethanol and DI water respectively and drying with $N_{2}$ gas. $2 \mathrm{mM}$ EDC and 5 $\mathrm{mM}$ NHS solution mixture was incubated for $50 \mathrm{~min}$ on the electrode surface at RT to activate the carboxylic acid group of SAM. The electrodes were then washed twice with PBS. $20 \mu \mathrm{L}$ of PBS containing $5 \mathrm{mg} / \mathrm{mL}$ GOx was dispensed onto the electrode, incubated for overnight at $4{ }^{\circ} \mathrm{C}$. The electrodes were then washed twice with PBS and stored at $4{ }^{\circ} \mathrm{C}$. The illustration of different steps in the immobilization of glucose on the PCB electrode is illustrated in Fig. 4.

Cleaned gold-plated electrodes were exploited as the anode electrode, by covalently immobilizing glucose oxidase. 50 $\mathrm{nm}$ platinum was sputter deposited on the cathode electrodes (power: $20 \mathrm{~W}$ power, base pressure: $9.310^{-6} \mathrm{mBar}$, process pressure: $6.3 \times 10^{-3} \mathrm{mBar}$ at a rate of $1.1 \mathrm{~A}^{\circ} / \mathrm{s}$ ). Glucose stock solutions was serially diluted in Phosphate Buffer Solution (PBS) and used for electrochemical measurements.

\section{Experimental Results}

Glucose solutions were prepared by diluting D-(+)- Glucose in PBS. $70 \mu \mathrm{L}$ of each sample was pipetted on the anode and cathode electrodes and the respective polarisation curves (plot of cell potential against current density) were recorded to assess fuel cell performance. An average of $0.123 \mathrm{~V}$ open circuit potential was recorded for our fuel cell structures. The polarisation curves were then used to calculate the power that the fuel cell will generate given a concentration of the analyte
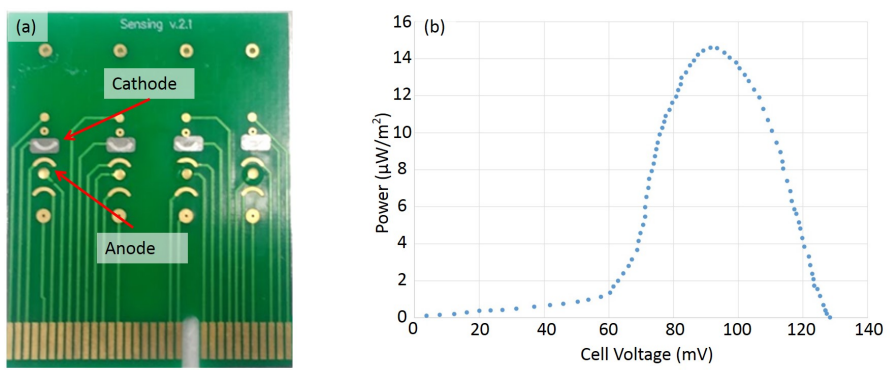

Fig. 5. (a) PCB fuel cell electrodes following the Pt deposition of the cathode, (b) Typical power curve obtained from PCB fuel cell with $100 \mathrm{mM}$ glucose stock solution.

(Power=current $\times$ Voltage), plotting the power against voltage as shown in Fig. 5.

\section{Circuit DESIGN}

The sensor patch consists of four main circuit blocks as shown in Fig. 6. The first part is responsible for interfacing with the sensor to read the glucose level and to monitor the sensor performance. For sensor interface, LMP9100 from TI is used. LMP9100 is a low power programmable analog front end for a sensor that generates an output voltage proportional to the cell current. The second part is an impedance spectroscopy chip (AD5933) to measure the sensor impedance and the provision of sensor self-calibration embedded onto the device.

The third part is the energy harvesting of the patch. This part of the circuit is to harvest energy from the biofuel cell to drive the system. To harvest energy, bq25570 from TI is used. The bq25570 is an integrated nano-power management for energy harvesting and can harvest energy from a source with voltage as low as $0.1 \mathrm{~V}$. On the other hand, it can supply current up to $110 \mathrm{~mA}$.

Finally, the microcontroller for system management and sending the data to the external interface. In this patch, low power microcontroller CC2640 chip is used which consists of a built-in BLE 5 which is suitable for low power applications because of it high speed and simple stack. The actual connection time is only a few ms, unlike traditional Bluetooth which require about $100 \mathrm{~ms}$. The microcontroller is running based on an event driven technique. During the idle mode, the microcontroller is in sleep mode, the sensor interface circuits

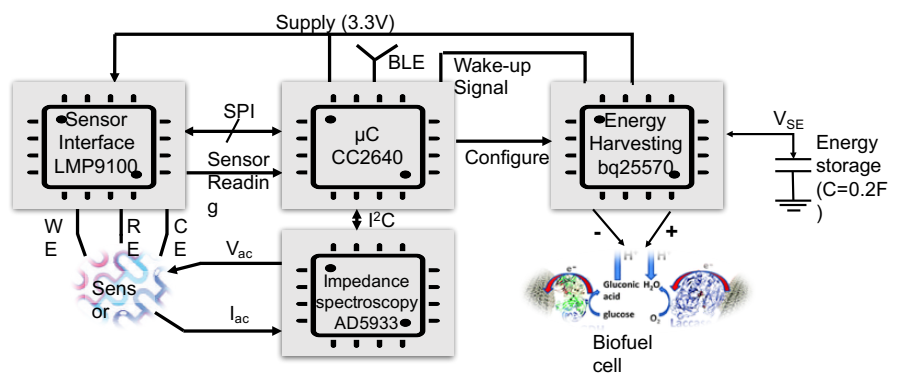

Fig. 6. Simplified circuit diagram of the glucose sensor patch. 


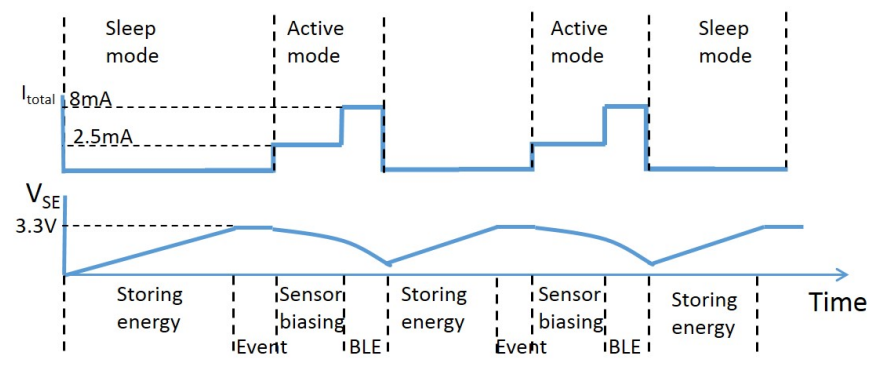

Fig. 7. Timing diagram for the different phases of operation for the system which shows charging happens when the system is in sleep mode and as the energy is stable the controller switch to the active mode to run the glucose measurement task

are disabled and the harvesting system is storing energy on a capacitor. When the stored energy reaches a threshold level, the energy management chip generates an event after a certain delay time. The purpose of the delay is to ensure that the stored energy will be enough and stable to run the system until finishing the task.

The generated event is used to wake up the microcontroller and subsequently the sensor interface circuit. The sensor requires almost one minute of continuous biasing before each measurement. After reading the sensor value, the microcontroller enables the BLE module to send the measured value outside. A simplified timing diagram of the system operation is depicted in Fig. 7.

The storage capacitor must be sized large enough to provide the required energy to run the system for $61 \mathrm{sec}$ ( $60 \mathrm{sec}$ for sensor biasing, 4msec for BLE transmission and 990msec for conversion and analysis). On average the required current is $2.5 \mathrm{~mA}$ for $60 \mathrm{sec}$ and $8 \mathrm{~mA}$ for $1 \mathrm{sec}$ due to the BLE. Hence, the estimated required capacitance is $0.2 \mathrm{~F}$ to guarantee that supply voltage will not drop below $2.8 \mathrm{~V}$. On the other hand, assuming no load (system in the sleep mode) in the beginning or during the charging cycle with an estimated harvester efficiency of $80 \%$, the estimated time to charge the capacitor is 1 hour. This means the system is able to send a glucose reading roughly every one hour. This is also assuming maximum power from the biofuel cell $(14 \mu \mathrm{W})$, and perfect impedance matching between the biofuel cell and the energy harvester chip. In reality the charging time may be further increased due to the voltage drop of the biofuel cell, the leakage current of the capacitor and the varying efficiency of the energy harvesting chip. This may be rectified by increasing the dimension of the anode and cathode electrodes or connecting few of them in parallel.

\section{ASSEMBLED DEVICE}

The whole system is fabricated on a $2.5 \times 3 \mathrm{~cm}$ double layer $\mathrm{PCB}$. The top layer is used for routing between the different parts of the circuit while the bottom layer is used for the fuel cell and the sensor. The whole circuit consumes $50 \mathrm{nA}$ during standby mode and $4 \mathrm{~mA}$ during the active mode. The PCB is currently undergoing post-processing for the development of the sensor and bio-fuel cell.

\section{CONCLUSIONS}

We presented the design of the different parts of a wearable patch for continuous glucose monitoring. This comprises of (i) a glucose sensor that is developed on PCB technology with demonstrated $150 \mathrm{nA} / \mathrm{mM}$ sensitivity to glucose; We discuss that a real-time automatic calibration might be possible based on monitoring the impedance of the sensor. (ii) A biofuel cell developed on PCB with measured maximum output power of $14 \mu \mathrm{W}$ from a fluid with $100 \mathrm{mM}$ glucose concentration; (iii) an instrumentation electronics for amperometric measurement of the sensor and energy and data management which consumes $50 \mathrm{nA}$ during standby mode and $4 \mathrm{~mA}$ during the active mode.

\section{ACKNOWLEDGEMENTS}

The project is supported by EPSRC eFutures Early Career Researcher's grant. The authors wish to acknowledge the financial support of British Council (Newton Fund Institutional Links, UK-Turkey project: 336872) and also thank the Spirit Circuits Group and Lyncolec Ltd for their collaboration in manufacturing the prototypes. S. Ghoreishizadeh would like to acknowledge the support of the Imperial College Junior Research Fellowship scheme.

\section{REFERENCES}

[1] L. Sydorchuk, K. Gumenna, O. Andriyets, A. Sydorchuk, and O. Bodnarjuk, "The pharma innovation-journal."

[2] C. Gonzalez-Solino and M. D. Lorenzo, "Enzymatic fuel cells: Towards self-powered implantable and wearable diagnostics," Biosensors, vol. 8, no. 1, p. 11, 2018.

[3] K. MacVittie and E. Katz, "Self-powered electrochemical memristor based on a biofuel cell-towards memristors integrated with biocomputing systems," Chemical Communications, vol. 50, no. 37, pp. 4816$4819,2014$.

[4] A. Zebda, S. Cosnier, J.-P. Alcaraz, M. Holzinger, A. Le Goff, C. Gondran, F. Boucher, F. Giroud, K. Gorgy, H. Lamraoui et al., "Single glucose biofuel cells implanted in rats power electronic devices," Scientific reports, vol. 3, 2013.

[5] A. F. Yeknami, X. Wang, S. Imani, A. Nikoofard, I. Jeerapan, J. Wang, and P. P. Mercier, "A 0.3v biofuel-cell-powered glucose/lactate biosensing system employing a 180nw 64db snr passive sigma-delta adc and a 920mhz wireless transmitter," in 2018 IEEE International Solid - State Circuits Conference - (ISSCC), 2018, pp. 284-286.

[6] D. R. in Children Network (DirecNet) Study Group, "Evaluation of factors affecting cgms calibration," Diabetes technology \& therapeutics, vol. 8, no. 3, pp. 318-325, 2006.

[7] A. B. Amar, A. B. Kouki, and H. Cao, "Power approaches for implantable medical devices," Sensors, vol. 15, no. 11, pp. 28 889-28914, 2015.

[8] G. Rovere, S. Fateh, and L. Benini, "A $2.2 \mu \mathrm{w}$ cognitive always-on wake-up circuit for event-driven duty-cycling of iot sensor nodes," IEEE Journal on Emerging and Selected Topics in Circuits and Systems, 2018.

[9] G. Dutta1, A. Regoutz, and D. Moschou1, "Commercially fabricated printed circuit board sensing electrodes for biomarker electrochemical detection: The importance of electrode surface characteristics in sensor performance," in Eurosensors 2018 Conference, Sep. 2018.

[10] S. S. Ghoreishizadeh, X. Zhang, S. Sharma, and P. Georgiou, "Study of electrochemical impedance of a continuous glucose monitoring sensor and its correlation with sensor performance," IEEE Sensors Letters, vol. 2, no. 1, pp. 1-4, 2018. 